Conclusions: Using an objective computerized technique, our study demonstrated a higher fall risk in patients with KOA than in healthy individuals. This higher risk was shown even in the early radiographic phases of the disease related to age, pain, and dysfunction. An understanding of factors on postural control seems to be critical in successful fall prevention in these patients.

Disclosure of Interest: None declared

DOI: 10.1136/annrheumdis-2017-eular.3072

\section{AB0810 CORRELATION BETWEEN KNEE EXTENSOR MUSCLE STRENGTH AND GAIT ENDURANCE AFTER TOTAL KNEE ARTHROPLASTY}

M.J. Suh, B.R. Kim. Jeju National University Hospital, Jeju, Korea, Republic Of

Objectives: This study was undertaken to identify the relationship of objective physical performance and gait endurance after 1 month after total knee arthroplasty.

Methods: One-hundred ninety-five patients ( 32 males and 163 females; average age $72.6 \pm 6.1$ years) who underwent a primary total knee arthroplasty (TKA). Patients completed 6-minute walk test (6MWT) to assess gait endurance. Additional physical performance test including timed up and go (TUG) test, timed Stair Climbing Test (SCT), instrumental gait analysis for spatio-temporal parameters, and isometric knee flexor and extensor strength of the surgical and nonsurgical knees 1 month after TKA were measured. To evaluate self-reported physical function, self-reported disease-specific physical function measured by using the Western Ontario McMaster Universities Osteoarthritis Index (WOMAC) and self-reported quality of life measured by using EuroQOL five dimensions (EQ-5D) questionnaire. Visual analog scale (VAS) of post-operative knee pain was recorded each patient.

Results: In the bivariate analyses, the postoperative gait endurance had a significant positive correlation with the postoperative gait speed $(r=0.48, p<0.001)$, cadence $(r=0.40, p<0.001)$, stride length $(r=0.58, p<0.001)$, postoperative peak torque (PT) extensor of surgical knee $(r=0.36, p<0.001)$, postoperative peak torque (PT) flexor of surgical knee $(r=0.25, p<0.001)$, postoperative peak torque (PT) extensor of non-surgical knee $(r=0.49, p<0.001)$, postoperative peak torque (PT) flexor of non-surgical knee $(r=0.34, p<0.001)$, EQ5D $(r=0.33, p<0.001)$ and a significant negative correlation with TUG $(r=-0.46, p<0.001)$, SCT-ascent $(r=-$ $0.63, p<0.001)$, SCT-descent $(r=-0.68, p<0.001)$, WOMAC pain score $(r=-0.29$, $p<0.001)$, WOMAC stiffness score $(r=-0.35, p<0.001)$, WOMAC function score $(r=-0.43, p<0.001)$

In the linear regression analyses, the postoperative Visual analog scale (VAS) $(\beta=-6.91, p=0.03)$, peak torque (PT) extensor of surgical knee $(\beta=0.56, p=0.04)$ and peak torque (PT) extensor of non-surgical knee $(\beta=0.77, p<0.001)$ were a factors predictive of the postoperative 6MWT.

\begin{tabular}{|c|c|}
\hline & $\begin{array}{c}\text { Comelation coefificients (f) } \\
\text { SAWT }\end{array}$ \\
\hline TUG (sec) & $-0.46^{\prime}$ \\
\hline \multicolumn{2}{|l|}{$\operatorname{sct}($ sec) } \\
\hline Ascent (sא) & $-0.63^{*}$ \\
\hline Descent (BeE) & $-0.68^{\circ}$ \\
\hline \multicolumn{2}{|l|}{ Gsit parameter } \\
\hline Gait Speed (m sec) & $0.48^{\circ}$ \\
\hline Cadence (steps $/ \min$ ) & $0.38^{\circ}$ \\
\hline Stride lenght (cm) & $0.23^{+}$ \\
\hline Gait cycle duration (ste) & -0.03 \\
\hline Stunce phase duration (\% of git cycle) & -0.11 \\
\hline Swing phase duration (\% of gait cycle) & 0.11 \\
\hline Double suppond duration (\% of gat cycle) & -0.02 \\
\hline Single suppont duration (\% of gait cycle) & -0.01 \\
\hline \multicolumn{2}{|l|}{ 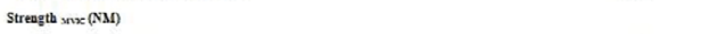 } \\
\hline PT extensor of furgeal knee (N. $\left.m \cdot k g^{\prime} B W\right)$ & $0.36^{\circ}$ \\
\hline PT flexor of surgical knee (N-m/kg $\left.g^{\prime} B W\right)$ & $0.25^{\prime}$ \\
\hline PT extensos of non-surgical knee (N-m:kg $\mathrm{BW})$ & $0.49^{\circ}$ \\
\hline PT flex or of ron-surgical knee (N.m:kg $B W$ & $0.34^{*}$ \\
\hline Deficit of extensor (\%) & 0.12 \\
\hline Deficit of Alexor (\%) & 0.13 \\
\hline \multicolumn{2}{|l|}{ ROM (degrees) } \\
\hline Knee fexion () & 0.08 \\
\hline $\mathrm{EQ}-5 \mathrm{D}$ & $0.33^{\circ}$ \\
\hline \multicolumn{2}{|l|}{ WOMAC } \\
\hline Pain & $-0.29^{\circ}$ \\
\hline Stiffess & $-0.35^{+}$ \\
\hline Function & $-0.43^{+}$ \\
\hline \multicolumn{2}{|l|}{ Valves sepresent correlation coefficients(s) } \\
\hline \multicolumn{2}{|l|}{-peos.05. $T p<0.01$} \\
\hline
\end{tabular}

Conclusions: This study demonstrated that quadriceps muscle strength significantly influenced on gait endurance 1 month after TKA. Using variables measured after surgery, it may be possible to predict with good accuracy for postoperative gait speed. In addition, these results could be of importance in determining effective rehabilitation strategies focusing on resistance training for improvement of gait speed early after TKA.

Disclosure of Interest: None declared

DOI: 10.1136/annrheumdis-2017-eular.1043

\section{AB0811 HYALURONIC ACID INTRA-ARTICULAR INJECTION VERSUS ORAL ATORVASTATIN IN THE TREATMENT OF KNEE OSTEOARTHRITIS}

M. Jokar, Z. Mirfeizi, K. Hashemzadeh, H. Zarei. Mashhad University of Medical Sciences, Mashhad, Iran, Islamic Republic Of

Background: Osteoarthritis is a degenerative joint disorder of articular cartilage and is the most common type of arthritis in the elderly. Some studies have suggested that Hyaluronic acid $^{1}$ and atorvastatin ${ }^{2}$ may have a role in the treatment of osteoarthritis.

Objectives: The purpose of the present study was to investigate and compare the potential effects of Hyaluronic acid and Atorvastatin on the symptoms of knee osteoarthritis.

Methods: We conducted an open, randomized, parallel study on patients with knee osteoarthritis. Eligible patients were those who met the ACR criteria of primary knee osteoarthritis, radiologically ascertained grade I or II of knee osteoarthritis on the Kellgren-Lawrence scale, had a pain score $\geq 60$ (maximum 100 ) and age 50-70 years. Patients are not eligible if they had secondary osteoarthritis, systemic disease like diabetes, history of inflammatory arthritis, sever knee deformity (varus or valgus $>50$ degree), history of coagolopathy, sever cardiovascular disease, and active infections. Patients were enrolled from outpatient clinic of Imam Reza Hospital, Mashhad, Iran. We divided them randomly into two groups; Group $1(\mathrm{n}=35)$ received intra-articular Hyaluronic acid each week for three weeks, and group $2(n=35)$ received oral atorvastatin 40 $\mathrm{mg} /$ day orally for six months. Symptoms were assessed by the Western Ontario and McMaster Universities Osteoarthritis Index (WOMAC) at baseline and every month up to 6 months.

Results: Twenty eight patients (40\%) were male and 42 patients (60\%) were female. There was not a significant difference between 2 groups regard to sex $(\mathrm{P}=0.626)$. Mean age of patients was $57.9 \pm 1.1$ years. Groups mean age did not differ significantly $(P=0.710)$. The pain and function scores were significantly decreased only in the Hyaluronic acid group in the second month $(P<0.001)$. There was not any significant improvement in any group in the next months (figure $1)$.

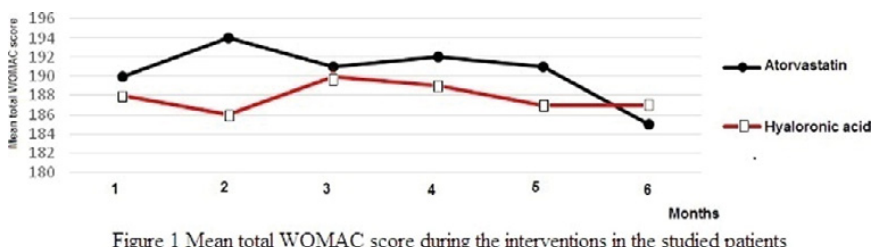

Conclusions: Intra articular Hyaluronic acid improved the pain and function of patients with knee osteoarthritis in the second months after injection. Atorvastatin did not have any effect on the knee osteoarthritis symptoms during 6 months.

References:

[1] Petrella RJ, Petrella M. A prospective, randomized, double-blind, placebo controlled study to evaluate the efficacy of intraarticular hyaluronic acid for osteoarthritis of the knee. J Rheumatol 2006; 33:951.

[2] Pathak NN, Lingaraju MC, Balaganur V, Kant V, More AS, Kumar D, Kumar D, Tandan SK. Anti-inflammatory and chondroprotective effects of atorvastatin in a cartilage explant model of osteoarthritis. Inflamm Res. 2015 Apr;64(34):161-9.

Disclosure of Interest: None declared

DOI: 10.1136/annrheumdis-2017-eular.2042

\section{AB0812 DIFFERENTIATED APPROACH TO THE TREATMENT OF OSTEOARTHRITIS WITH COMORBIDITIES}

N. Shuba, A. Krylova, T. Khambir, A. Pilipenko, T. Voronova. Shupyk National Medical Academy of Postgraduate Education, Kyiv, Ukraine

Background: A significant prevalence of osteoarthritis (OA), the most disability joint disease in the world, which is important in the search for the new treatment. Analysis of modern therapy of OA was the reason for research efficacy of NSAIDs and SYSADOA on biochemical, inflammatory and immunological signs in the treatment of $O A$.

Objectives: Differentiated approach to the treatment of OA depending of presence of hyperuricemia.

Methods: 176 patients ( 144 women, 32 men) was exanimated, aged $(59,71 \pm 0,86)$ years with confirmed radiographic OA according to Kellgren and Lawrence scale. Division into groups was performed depending on treatment. For 14 days group 1 $(n=30)$ received nimesulide $100 \mathrm{mg}$ twice daily, and group $2(n=30)$ - meloxicam 
$15 \mathrm{mg} 1$ per day. For 6 months 3rd group $(\mathrm{n}=54)$ received diacerein $50 \mathrm{mg}$ twice a day and the 4 th group ( $n=32)$ - chondroitine sulfate (CS) $500 \mathrm{mg}$ twice daily. 5th group $(n=30)$ received glucosamine sulfate (GS) $400 \mathrm{mg} 3$ times per week for 1.5 months. It was estimated dynamics of VAS, WOMAC, Lequesne, uric acid (UA), CRP, IL-1 $\beta$, IGF-1, NO, ESR, biochemical indicators. Statistical analysis - SPSS Statistics.

Results: Nimesulide compared to meloxicam found more effective for WOMAC index, the reduction of pain, stiffness and functional failure $53.93 \%$ vs $37.3 \%$, $29.7 \%$ vs $13.5 \%, 41.6 \%$ vs $26.6 \%$ respectively. Only nimesulide decreased NO by $40.2 \%$, and showed a decrease trend of UA from $375,33 \pm 24,6$ to $321,57 \pm 19,6 \mathrm{mkmol} / \mathrm{l}$ while meloxicam had no effect on their levels. In $60 \%$ of patients with OA was found hyperuricemia with nephrolithiasis. Was showed the relation between the severity of OA and the presence of hyperuricemia by VAS, Lequesne, WOMAC, lesions more number joints, higher stage of X-ray progress. Diacerein, CS and GS showed clinical effect on pain reduction by VAS, Lequesne, WOMAC $(p<0.05)$, respectively. Was showed lower levels of CRP, IL-1, NO during treatment by diacerein (CRP from 5,6 (IQR: $3,6-8,0$ ) to 3,0 (IQR: $1,95-4,3$ ) mg/l, IL-1 from 19,47 (IQR: 15,22-23,81) to 12,0 (IQR: 9,0-16,2) pg/ml, NO of 4,09 (IQR: 3,0-4,19) to 2,2 (IQR: 1,0-3,44) mmol/l), CS (CRP from 6,0 (IQR: 3,25-9,0) to 3,10 (IQR: 1,81-6,0) $\mathrm{mg} / \mathrm{l}$, IL-1 20,0 (IQR: 11,5-26,52) pg/ml to 16,7 (IQR: 9,80-18,62) pg/ml, NO of 3,0 (IQR: 2,05-4,00) to 2,0 (IQR: $2,0-3,0) \mathrm{mmol} / \mathrm{l})$ and GS (CRP from 6,0 (IQR: 4,0-8,45) to 4,0 (IQR: 4,0-6,0) mg/l, IL-1 from 4,69 (IQR: 2,5-7,59) to 2,52 (IQR: $1,75-3,94) \mathrm{pg} / \mathrm{ml}$, NO 3,28 (IQR: 2,02-4,79) to 2,37 (IQR: 1,59-3,03) mmol/I) $(p<0,05)$. Diacerein reduced UA from 415,5 (IQR: $347,0-452,5)$ to 374,0 (IQR: $318,75-423,5) \mathrm{mkmol} / \mathrm{l}(\mathrm{p}=0.001)$ and glucose from 5,1 (IQR: $4,8-5,8) \mathrm{mmol} / \mathrm{l}$ to 4,88 (IQR: $4,00-5,40) \mathrm{mmol} / \mathrm{l}(\mathrm{p}=0.0001)$. CS in patients with hyperuricemia $(p=0.001)$ increased the level of $U A$, which reduced the efficacy of CS, but without hyperuricemia didn't influence the level of UA and efficacy of CS.

Conclusions: Was showed that nimesulide is more effective than meloxicam during first 14 days, and reduces NO, an important proinflammatory factor. The presence of hyperuricemia in $60 \%$ patients with $\mathrm{OA}$, resulted in a more severe $\mathrm{OA}$. Diacerein decreases UA and glucose, given an advantage in treatment these patients, which isn't presented with CS (which increased UA).

References:

[1] National Clinical Guideline Centre. Osteoarthritis. Clinical guideline CG177; 2014.

[2] Hyperuricemia and the risk for subclinical coronary atherosclerosis - data from a prospective observational cohort study. Krishnan E. et al. Arthritis Res. Ther. 2011;13(2):66.

Disclosure of Interest: None declared

DOI: 10.1136/annrheumdis-2017-eular.3407

\section{AB0813 PROPOSAL FOR THE CONSTITUTION IMPLEMENTATION AND DEVELOPMENT OF THE PROGRAM OF CENTERS OF EXCELLENCE IN OSTEOARTHRITIS IN LATIN AMERICA}

P. Santos-Moreno ${ }^{1}$, R. Arape ${ }^{2}$, R. Chacon ${ }^{2}$, L. Franco ${ }^{2}$, H. Riera ${ }^{2}$, C. Caballero ${ }^{3}$, M. Quintero ${ }^{2}{ }^{1}$ Rheumatology, Biomab, Center for Rheumatoid Arthritis, Bogota, Bogota, Colombia; ${ }^{2}$ Rheumatology, Center for Osteoarthritis, Caracas, Caracas, Venezuela, Bolivarian Republic Of; ${ }^{3}$ Rheumatology, Universidad del Norte, Barranquilla, Colombia

Background: Osteoarthritis $(\mathrm{OA})$ is the most prevalent disease in the Americas, with an estimated prevalence of $5-10 \%$ of the population. These patients have difficulties accessing the clinic, as well as poorly successful therapeutic programs. We plan to develop a program of Centers of Excellence (CoE) in OA, to meet and respond to these needs, generate data and local research applicable to our health systems.

Objectives: In a first phase: To create OA pilot centers in Venezuela and Colombia under the CoE scheme, defined in REAL-PANLAR project (Panamerican league of associations for rheumatology project for implementation of $\mathrm{CoE}$ in Rheumatoid Arthritis). In a second phase under the auspices of PANLAR to create the Pan American Network of Centers of Excellence in OA (CEOA).

Methods: A systematic review of the literature in global and regional databases (Pubmed, Medline, Scopus, Lilacs) was conducted and through a Delphi-modified methodology and a consensus of experts, naming a coordinator by subject, we established the conceptual bases and defined OA Center of Excellence.

Results: The group proposes: 1 . Clinical detection and early diagnosis of OA, discarding false positives and diagnosing early $\mathrm{OA} ; 2$. Model of patient-centered and multidisciplinary care: frequency of care are to be established according to the conditions of the patient and the multidisciplinary team. 3. Implementation of a specific clinical register of OA with indexes such as WOMAC, Lequesne and also EuroQol and definition of socioeconomic characteristics. 4. Paraclinics and Imaging: Each patient will be given a general laboratory study at admission and then every two years including a metabolic profile. Conventional Rx of hands, hips, knees or lumbosacral column depending on the location and then annual or depending on the evolution. Ultrasound, upon admission to the program, six month later, if synovitis was detected, for redefining the treatment and then annually for evaluation of the articulatory damage; CT and MRI when deemed necessary (OMERACT) 5. Definition of the disease management program in axes: treatment protocol of pain with sequential treatment lines, DMOADs, viscosupplementation, Glucosamine + Chondroitin, Strontium Ranelate, Piascledin; Alternative Medicine and Surgical Treatment 6. Establishment of a management program that includes Structure, Process and Outcome Indicators to define the CEOA as Standard, Optimal and Model. 7. Education modules for patients and families; Continuing Medical Education for all members of the multidisciplinary team; Generation of research in the areas above mentioned.

Conclusions: The unmet needs in OA determine the implementation of units of CEOAs, to promote management knowledge, and define T2T strategies, in order to improve clinical outcomes, reducing disability and consequently economic and social costs.

\section{References:}

[1] REAL-PANLAR Project for the Implementation and Accreditation of Centers of Excellence in Rheumatoid Arthritis in Latin America: A Paper From REALPANLAR Group on Improvement of Rheumatoid Arthritis Care in Latin America Establishing Centers of Excellence. Santos-Moreno P, Galarza-Maldonado C, Caballero-Uribe CV, et al. J Clin Rheumatol. 2015 Jun;21(4):175-80.

Disclosure of Interest: None declared

DOI: 10.1136/annrheumdis-2017-eular.4557

\section{AB0814 MEDIAL SHIFT OF THE TIBIAL ARTICULAR SURFACE SHOULD BE TAKEN INTO ACCOUNT FOR ONE FACTOR OF MEDIAL OSTEOARTHRITIS OF THE KNEE}

R. Nagamine. Center of Artificial Joint and Rheumatism, Fukuoka Tokushukai Medical Center, Kasuga, Japan

Background: Varus knee is one factor of medial osteoarthritis of the knee. According to the concept of the constitutional varus ${ }^{1)}$, the bone growth disturbance at the growth plate in the medial proximal metaphysis of the tibia results in proximal tibia vara. In this situation, the tibia is bent at the proximal metaphysis and the tibial articular surface (TAS) may be shift medially. The medial shift of the TAS will increase the mechanical loading in the medial side of the knee. The medial shift of TAS will also influence the value of the Hip-knee-ankle (HKA) angle because the tibial plafond that is the end point of the mechanical axis (MA) of the tibia will shift laterally.

Objectives: The purpose of this study was to assess the extent of the medial shift of TAS in knees with medial osteoarthritis, and to assess the effect of this medial shift of TAS on the value of HKA angle.

Methods: This study consists of 116 knees with medial osteoarthritis. The mean age was 75.3 years old. The mean standing femorotibial angle (FTA: lateral angle between femoral and tibial anatomical axes) was $183.6^{\circ}$. The anatomical axis (AA) was the central line of the femoral and the tibial shaft. On the anteroposterior view radiograph of the tibia, $A A, M A$ and tibial plateau tangent were drawn. MA is the line between the center of the tibial spines notch and the center of the tibial plafond. Two angle parameters and two distance parameters were measured. Those are angle between $A A$ and MA (Angle AA-MA) (the value was positive when MA located medial to AA), angle between the tibial plateau tangent and the line perpendicular to AA (Angle plateau), distance from AA to the center of the tibial spines notch on the tibial plateau (Distance AA-MA) (the value was positive when Point $M$ located medial to $A A)$, and the length of $M A$.

Results: The mean $( \pm S D)$ Angle AA-MA and Angle plateau was $1.0^{\circ} \pm 0.6^{\circ}$ and $8.2^{\circ} \pm 2.9^{\circ}$, respectively. The mean Distance AA-MA and length of MA was $5.6 \pm 3.4 \mathrm{~mm}$ and $347.3 \pm 3.4 \mathrm{~mm}$, respectively. Figure 1 shows the relationship between Angle Plateau and Distance AA-MA. The correlation coefficient between two parameters was 0.62 . The more proximal tibia had varus deformity, the more the tibial articular surface shifted medially. The maximum Distance AA-MA was $16.1 \mathrm{~mm}$. In this case, HKA angle underestimated varus deformity up to $3^{\circ}$.

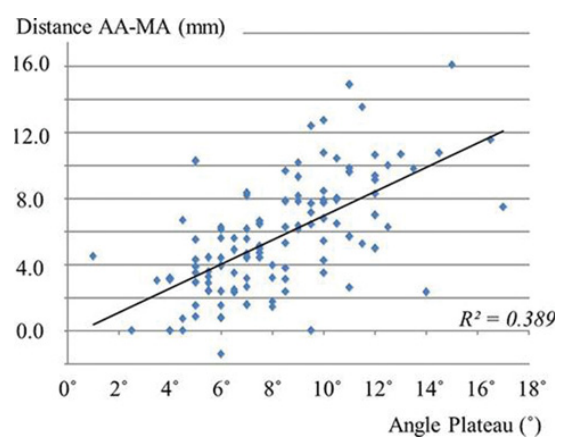

Conclusions: The knees with proximal tibia vara have medial shift of the tibial articular surface. There are three factors to influence the evaluations of the medial osteoarthritis of the knees. Firstly, varus knees have higher loading condition in medial compartment because of proximal tibia vara and medial shift of the tibial articular surface. Secondly, HKA angle under-estimates varus deformity in knees with medial shift of the tibial articular surface. Thirdly, FTA cannot reveal the exact loading condition, either. Even with the same FTA, it is clear that loading in the medial compartment is larger in knees with medial shift of the tibial articular surface compared with knees with simple medial osteoarthritis. The medial shift of the tibial articular surface should be taken into account for one factor of medial osteoarthritis of the knee. 